\title{
Combining CSP and MPC for the Operational Control of Water Networks Application to the Richmond Case Study ${ }^{\star}$
}

\author{
Cong Cong Sun * Vicenç Puig * Gabriela Cembrano ${ }^{*, * *}$ \\ *Advanced Control Systems Group at the Institut de Robòtica i Informàtica \\ Industrial (CSIC-UPC), Llorens i Artigas, 4-6, 08028 Barcelona, (Spain). \\ Email: vicenc.puig@upc.edu.Tel: +34688041855 \\ ** CETaqua, Water Technology Centre at Esplugues 75, 08940, Cornellà de \\ Llobregat, Barcelona
}

\begin{abstract}
This paper presents the combination of linear Model Predictive Control (MPC) with Constraint Satisfaction Problem (CSP) for the operational control of drinking water networks. The methodology has been divided into two functional layers: First, a CSP algorithm is used to transfer non-linear pressure equations of drinking water networks (DWNs) into linear constraints, which can enclose feasible solution of the hydraulic non-linear problem during the optimizing process. Then, a linear MPC with added linear constraints is solved to generate control strategies which consider control objectives. Finally, the proposed approach is simulated using Epanet to represent the real DWNs. PLIO, which is a generic operational tool for controlling water networks that uses non-linear MPC, is used for validation. The Richmond water system is used as a case study.
\end{abstract}

Keywords: MPC, CSP, Epanet, PLIO.

\section{INTRODUCTION}

DWNs are complex multiple-input and multiple-output systems with both deterministic and stochastic components which involve linear (flow model) as well as non-linear (pressure model) dynamics.

Management of both linear and non-linear problems typically rely on simplified network hydraulic model as described in Papageorgiou (1983). Other authors employ discrete dynamic programming as presented in Zessler and Shamir (1989), which is only applicable to small networks. There exist tools like PLIO in Cembrano et al. (2011), which is able to solve both linear and non-linear MPC problems with a gradient-based optimization being only able to guarantee local optimal solutions.

MPC is a well-established class of advanced control methods as explained in Rawlings and Mayne (2009). In Ocampo-Martinez et al. (2012), MPC has been successfully applied for controlling and optimizing linear flow model of DWNs. But when the pressure model is considered, because of the introduction of non-linear functions, the computation complexity of MPC will increase considerably with the size of the network. Besides, when using non-linear MPC, convergence to the global minimum cannot be guaranteed as described in AbdelMeguid (2011).

This paper mainly provides a methodology for solving large non-linear problems of DWNs using linear MPC with the cooperation of CSP. As shown in Fig. 1, the whole control methodology works in a two-layer structure: CSP is the first step of this methodology and behaves as the upper layer for producing

\footnotetext{
* This research has been partially funded by the DGR of Generalitat de Catalunya (SAC group Ref. 2009/SGR/1491), Doctorat Industrial 2013-DI-041 and by EFFINET.
}

linear constraints for MPC; while MPC optimization behaves as the second layer and also connects and communicates with the upper layer CSP.

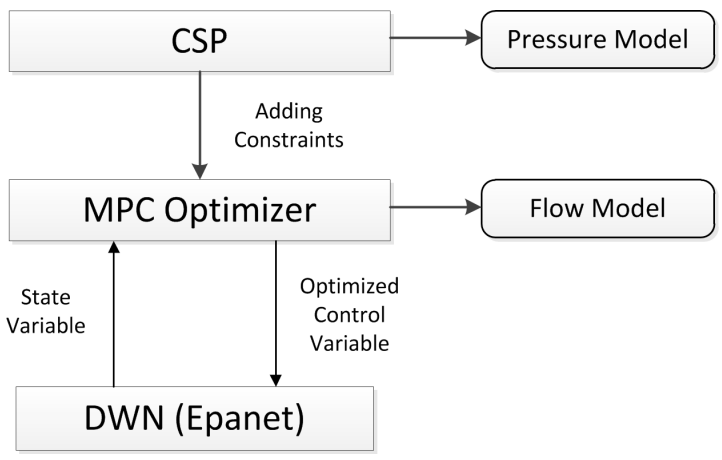

Figure 1. The multi-layer control scheme

The remainder of the paper is organized as follows. The controloriented modelling methodology is obtained in Section 2. Then, in Section 3, the operational control problem statement is introduced. In Section 4, the definition of CSP and also the proposed CSP-MPC control scheme are explained in detail. Section 5 summarized the validation results and finally, Section 6 contains the conclusions and future work ideas.

\section{CONTROL-ORIENTED MODELLING METHODOLOGY}

The DWNs can be considered as composed of a set of constitutive elements, which are presented and discussed separately below modelling flow and pressure, respectively. 


\subsection{Flow Model}

Reservoirs and Tanks. Water reservoirs and tanks provide the entire network with the water storage capacity. The mass balance expression of these storage elements relating the stored volume $v$, the manipulated inflows $q_{\text {in }}^{i, j}$ and outflows $q_{\text {out }}^{i, l}$ for the $i^{t h}$ storage element can be written as the discrete-time difference equation

$$
v_{i}(k+1)=v_{i}(k)+\Delta t\left(\sum_{j} q_{\mathrm{in}}^{i, j}(k)-\sum_{l} q_{\mathrm{out}}^{i, l}(k)\right),
$$

where $\Delta t$ is the sampling time and $k$ denotes the discretetime instant. The physical constraint related to the range of admissible water in the $i^{\text {th }}$ storage element is expressed as

$$
\underline{v}_{i} \leq v_{i}(k) \leq \bar{v}_{i}, \quad \text { for all } k,
$$

where $v_{i}$ and $\bar{v}_{i}$ denote the minimum and the maximum admissible storage capacity, respectively.

Actuators. Two types of control actuators are considered: valves/gates and pumps. The manipulated flows through the actuators represent the manipulated variables, denoted as $q_{u}$. Both pumps and valves/gates have lower and upper physical limits, which are taken into account as system constraints. As in (2), they are expressed as

$$
\underline{q u}_{i} \leq q_{u i}(k) \leq \overline{q_{u}}, \quad \text { for all } k,
$$

where $q_{u_{i}}$ and $\overline{q_{u}}$ denote the minimum and the maximum flow capacity, respectively.

Nodes. The nodes represent mass balance relations, being modelled as equality constraints related to inflows and outflows. The expression of the mass conservation in these nodes can be written as

$$
\sum_{j} q_{\text {in }}^{i, j}(k)=\sum_{h} q_{\text {out }}^{i, h}(k) .
$$

Demand Sectors. Demand and irrigation sector represents the water demand made by the network users of a certain physical area. The demand forecasting algorithm typically uses a twolevel scheme composed by (i) a time-series model to represent the daily aggregate flow values, and (ii) a set of different daily flow demand patterns according to the day type to cater for different consumption during the weekends and holidays periods.

\subsection{Pressure Model}

When combined with a pressure model, the flow model presented in the previous section should be extended using the non-linear relation between flow and pressure, which appears at pipes, valves, pumps and tanks as in Brdys and Ulanicki (1994).

Pipes. The Chezy-Manning model is one of the various widely used models to describe pressure loss between two nodes $i$ and $j$ linked by a pipe:

$$
g(q)=h_{i}-h_{j}=g_{i j}\left(q_{i j}\right)=R_{i j} q_{i j}^{2}
$$

where

$$
R_{i j}=\left(10.29 \times L_{i j}\right) /\left(C_{i j}^{2} \times D_{i j}^{5.33}\right)
$$

and $L_{i j}, D_{i j}$ and $C_{i j}$ denote the pipe length, diameter and coefficient.
Pumps. Pumps introduce a positive increase of pressure between the suction node $s$ and the delivery node $d$. In the more general case that corresponds to variable speed pumps, the relation between the flow and the pressure increase is given by:

$g(q, u, s)=h_{d}-h_{s}= \begin{cases}W q^{2}+M q+N s^{2}, & \text { if } u \neq 0 \text { and } s \neq 0 \\ 0, & \text { otherwise }\end{cases}$

where $s$ is the pump speed and $u$ corresponds to the number of pumps that are turned on, $W, M$ and $N$ are pump specific coefficients.

Valves. These valves can be modelled as a pipe with controlled conductivity, that is

$$
g_{i j}\left(q_{i j}, v_{i j}\right)=G_{i j} R_{i j} q_{i j}^{2}
$$

where $R_{i j}$ is the pipe conductivity and $G_{i j}$ is the control variable that manipulates the valve opening from 0 (closed) to 1 (open).

Tanks. The pressure established by the $i^{\text {th }}$ tank is given by the following equation:

$$
h_{r i}(t)=\frac{v_{i}(t)}{S e c_{i}}+E_{i}
$$

where $S e c_{i}$ is the cross-sectional area of the tank and $E_{i}$ is the tank elevation.

\section{OPERATIONAL CONTROL PROBLEM STATEMENT}

The type of control in DWNs can mainly be separated into two categories: linear control for the flow model and non-linear control for the pressure model as in Brdys and Ulanicki (1994).

\subsection{MPC for Flow Control}

In the case of the flow control problem, the standard MPC problem based on the linear discrete-time prediction model is considered as in Maciejowski (2002):

$$
\begin{aligned}
x(k+1) & =A x(k)+B u(k), \\
y(k) & =C x(k),
\end{aligned}
$$

where $x(k) \in \mathbb{R}^{n_{x}}$ is state vector represents tank volume and $u(k) \in \mathbb{R}^{n_{u}}$ is vector of command variables of actuator flows at time step $k, y(k) \in \mathbb{R}^{n_{y}}$ is the vector of the measured outputs. Matrices $A$ and $B$ are obtained using flow modelling approach in Section 2.1.

We define an incidence matrix $\Lambda_{c}$ for junction nodes in order to write equation (4) in matrix form, where the element in the $i^{\text {th }}$ column and $j^{\text {th }}$ row of junction nodes incidence matrix $\Lambda_{c}$ is defined as:

$$
a_{i j}= \begin{cases}1 & \text { if flow of branch } i \text { enters node } j \\ 0 & \text { if branch } i \text { and node } j \text { are not connected } \\ -1 & \text { if flow of branch } i \text { leaves node } j\end{cases}
$$

Assuming one network has $n_{c}$ non-storage nodes and $b$ branches, thus, this incidence matrix are $n_{c}$ rows and $b$ columns.

A matrix form of equation (4) is as follows:

$$
\Lambda_{c} q=d
$$

where $q=\left(q_{1}, \ldots, q_{b}\right)^{T}$ is a vector of branch flows, $d$ denotes an augmented demand vector by zero components corresponding to non-loaded nodes.

Thus, the following basic optimization problem (BOP) has to be solved: 


\section{Problem 1:}

$$
\begin{array}{ll} 
& \min _{\left(u(0 \mid k), \cdots, u\left(H_{p-1} \mid k\right)\right)} J(k) \\
\text { s.t. } & x(i+1 \mid k)=A x(i \mid k)+B u(i \mid k), \quad i=1, \cdots, H_{p}, \\
& x(0 \mid k)=x_{k}, \\
& \Lambda_{c} u=d \\
& x_{\text {min }} \leq x(i \mid k) \leq x_{\text {max }}, \quad i=1, \cdots, H_{p}, \\
& u_{\text {min }} \leq u(i \mid k) \leq u_{\text {max }}, \quad i=0, \cdots, H_{p-1},
\end{array}
$$

and Problem 1 can be recast as a Quadratic Programming (QP) problem:

$$
\mathcal{U}^{*}(k) \triangleq\left[u^{*}(0 \mid k) \cdots u^{*}\left(H_{p}-1 \mid k\right)\right]^{T} \in \mathbb{R}^{H_{p} m \times 1}
$$

At each sampling time $k$, Problem 1 is solved for the given measured current state $x(k)$. Only the first optimal move $u^{*}(0 \mid k)$ of the optimal sequence $\mathcal{U}^{*}(k)$ is applied to the process:

$$
u_{M P C}(k)=u^{*}(0 \mid k)
$$

the remaining optimal moves are discarded and the optimization is repeated at time $k+1$.

\subsection{Operational Goals for Flow Control}

The main operational goals to be achieved in water distribution networks are:

- Cost reduction $\left(J_{\text {cost }}\right)$ : Minimize water cost during water supplying process.

- Operational safety $\left(J_{\text {safety }}\right)$ : Maintain appropriate water storage levels in tanks of the network for emergencyhandling.

- Control actions smoothness ( $\left.J_{\text {smoothness }}\right)$ : Smooth flow setpoint variations for sustainable process.

Above mentioned goals lead to the following function:

$$
\begin{aligned}
J & =J_{\text {safety }}+J_{\text {smothness }}+J_{\text {cost }} \\
& =\varepsilon_{\widetilde{x}}(k)^{\top} W_{\widetilde{x}} \varepsilon_{\widetilde{x}}(k)+\Delta \widetilde{u}(k)^{\top} W_{\widetilde{u}} \Delta \widetilde{u}(k) \\
& +W_{a}\left(a_{1}+a_{2}(k)\right) \widetilde{u}(k)
\end{aligned}
$$

where

$$
\begin{aligned}
\varepsilon_{\widetilde{x}}(k) & =\widetilde{x}(k)-\widetilde{x}_{r} \\
\widetilde{u} & =\Theta \Delta \widetilde{u}+\Pi \widetilde{u}(k-1) \\
\Delta \widetilde{u}(k) & =\widetilde{u}(k)-\widetilde{u}(k-1)
\end{aligned}
$$

vectors $a_{1}$ and $a_{2}$ contain the cost of water treatment and pumping, and $W_{\widetilde{x}}, W_{\widetilde{u}}, W_{a}$ are the related weights established by the water network managers.

\subsection{Nodal Model for Pressure Control}

After combining equation (9) with equation (1), tank dynamics both considering flow and pressure will be presented as:

$$
\left\{\begin{array}{l}
h_{r i}(t)=\frac{v_{i}(t)}{S e c_{i}}+E_{i} \\
v_{i}(k+1)=v_{i}(k)+\Delta t\left(\sum_{j} q_{\text {in }}^{j}(k)-\sum_{h} q_{\text {out }}^{h}(k)\right)
\end{array}\right.
$$

Considering a network with $n$ nodes and $b$ branches, the nodebranch matrix $\Lambda$ will have $n$ rows and $b$ columns. Consider element $b_{i j}$ in the $i^{\text {th }}$ row in the $j^{\text {th }}$ column as equation (11) holds.

For the sake of convenience, we will place the rows corresponding to the tank/reservoir nodes on the first $n_{r}$ position. The other rows correspond to the junction nodes. With the help of matrix
$\Lambda$ we can write the flow-head equations as the following vector equation:

$$
\Lambda^{T}\left[\begin{array}{c}
h_{r} \\
h
\end{array}\right]+G(q)=0
$$

where

- $h_{r}=\left(h_{r 1}, \cdots, h_{r, n_{r}}\right)^{T}$ heads of tank nodes

- $h=\left(h_{1}, \cdots, h_{n_{c}}\right)^{T}$ heads of junction nodes

- $q=\left(q_{1}, \cdots, q_{b}\right)^{T}$ branch flows

- $G(q)=\left(g_{1}\left(q_{1}\right), \cdots,-g_{i}\left(q_{i}, u_{i}\right), \cdots, g_{1}\left(q_{1}, v_{1}\right), \cdots,\right)^{T}$ functions defining flow-head relationships

This equation combined with equation (4) yields the nodal model:

$$
\left\{\begin{array}{l}
\Lambda_{c} q=d \\
\Lambda^{T}\left[\begin{array}{c}
h_{r} \\
h
\end{array}\right]+G(q)=0
\end{array}\right.
$$

\subsection{MPC for Pressure Control}

In the case of pressure control of DWNs, the MPC problem is defined as:

\section{Problem 2}

$$
\begin{array}{ll} 
& \min _{\left(u(0 \mid k), \cdots, u\left(H_{p-1} \mid k\right)\right)} J(k) \\
\text { s.t. } & x(i+1 \mid k)=A x(i \mid k)+B u(i \mid k), \quad i=1, \cdots, H_{p}, \\
& x(0 \mid k)=x_{k}, \\
& \Lambda_{c} u=d \\
& h_{r}(i \mid k)=\frac{x(i \mid k)}{S e c_{i}}+E_{i}
\end{array}
$$

and:

$$
\Lambda^{T}\left[\begin{array}{c}
h_{r} \\
h
\end{array}\right]+G(u)=0
$$

where:

$$
\begin{aligned}
& x_{\min } \leq x(i \mid k) \leq x_{\max }, \quad i=1, \cdots, H_{p}, \\
& u_{\min } \leq u(i \mid k) \leq u_{\max }, \quad i=0, \cdots, H_{p-1},
\end{aligned}
$$

As described above, MPC of pressure control is highly nonlinear because of added pressure constrains in equation (21), which adds complexity to the optimization problem for the large scale DWNs.

This paper proposes to use MPC integrated with CSP to solve the non-linear control problem of DWNs by including linear constraints that come from considering the pressure model.

\section{PROPOSED APPROACH}

\subsection{Overview of Scheme CSP-MPC}

The integration scheme of CSP (see below) and MPC for DWNs considering the pressure model is presented in Fig. 2, which shows that, the main principle of this proposed control scheme is transferring the constraints of the non-linear pressure model into linear constraints which may be tackled by the flow MPC model by using the CSP algorithm. The linear constraints produced by CSP will be combined with the initial constraints of linear MPC for DWNs as new constraints. With this scheme, Problem 2 which is non-linear MPC will be updated into linear MPC problem: 
Problem 3

$$
\begin{array}{ll} 
& \min _{\left(u(0 \mid k), \cdots, u\left(H_{p-1} \mid k\right)\right)} J(k) \\
\text { s.t. } & x(i+1 \mid k)=A x(i \mid k)+B u(i \mid k), \quad i=1, \cdots, H_{p}, \\
& x(0 \mid k)=x_{k}, \\
& \Lambda_{c} u=d
\end{array}
$$

where:

$$
\begin{aligned}
& x_{\text {min }}^{\prime} \leq x(i \mid k) \leq x_{\text {max }}^{\prime}, \\
& u_{\text {min }}^{\prime} \leq u(i \mid k) \leq u_{\text {max }}^{\prime}, \quad i=0, \cdots, H_{p},
\end{aligned}
$$

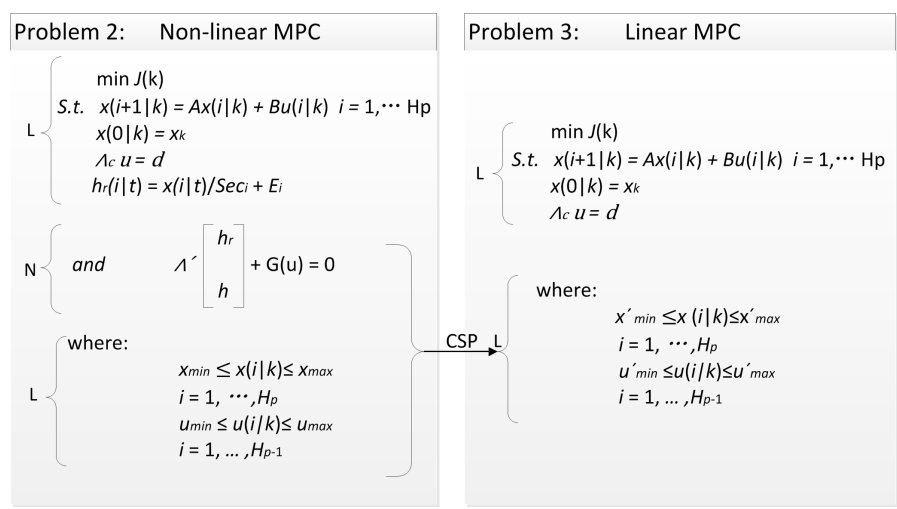

Figure 2. Working principle of CSP-MPC

The constraints $x_{\min }^{\prime}, x_{\text {max }}^{\prime}, u_{\text {min }}^{\prime}$ and $u_{\text {max }}^{\prime}$ will be will be obtained by solving a CSP problem.

\subsection{Definition of CSP}

A CSP is typically defined as the problem of finding any consistent labelling for a fixed set of variables satisfying all given constraints between these variables.

Normally a CSP consists of $n$ variables $v_{1}, v_{2}, \ldots, v_{n}$, whose values are taken from finite, discrete domains $D_{1}, D_{2}, \ldots D_{n}$, respectively, and a set of constraints on their values. A constraint is defined by a predicate. That is, the constraint $c_{k}\left(c_{k 1}, \ldots, c_{k j}\right)$ is a predicate that is defined on the Cartesian product $D_{k 1} \times$ $\ldots \times D_{k j}$. This predicate is true if the value assignment of these variables satisfies these constraints. Solving a CSP is equivalent to finding an assignment of values to all variables such that all constraints are satisfied as in Kumar (1998).

On sets, a CSP can be formulated as a 3-tuple $\mathcal{H}=(\mathcal{V}, \mathcal{D}, C)$, where

- $\mathcal{V}=\left\{v_{1}, \cdots, v_{n}\right\}$ is a finite set of variables.

- $\mathcal{D}=\left\{\mathcal{D}_{1}, \cdots, \mathcal{D}_{n}\right\}$ is the set of their domains.

- $C=\left\{c_{1}, \cdots, c_{n}\right\}$ is a finite set of constraints relating variables of $\mathcal{V}$.

Solving a CSP consists in finding all variable value assignments such that all constraints are satisfied. The variable value assignment $\left(\hat{z}_{1}, \cdots, \hat{z}_{n}\right) \in \mathcal{D}$ is a solution of $\mathcal{H}$ if all constraints in $C$ are satisfied. The set of all solution points of $\mathcal{H}$ is called the global solution set and denoted by $\mathcal{S}(\mathcal{H})$. The variable $v_{i} \in \mathcal{V}$ is consistent in $\mathcal{H}$ if and only if $\forall \hat{z}_{i} \in \mathcal{D}_{i}, \exists\left(\hat{z}_{1} \in \mathcal{D}_{1}, \cdots, \hat{z}_{n} \in\right.$ $\left.\mathcal{D}_{n}\right)$, such as $\left(\hat{z}_{i}, \cdots, \hat{z}_{n}\right) \in \mathcal{S}(\mathcal{H})$ in Tornil-Sin et al. (2014).

The solution of a CSP is said to be globally consistent if and only if every variable is consistent. A variable is locally consistent if and only if it is consistent with respect to all directly connected constraints. Thus, the solution of the CSP is said to be locally consistent if all variables are locally consistent. An algorithm for finding an approximation of the solution set of a CSP can be found in Jaulin et al. (2001).

\subsection{CSP-MPC Algorithm}

The algorithm of CSP-MPC is described as Algorithm 1. In every iteration time, this CSP algorithm will produce updated constraints equation (24) to Problem 2 by means of propagating the effect of non-linear constraints equation (21) into the operational constraints equation (22), which will be used for linear MPC to generate optimized control strategies.

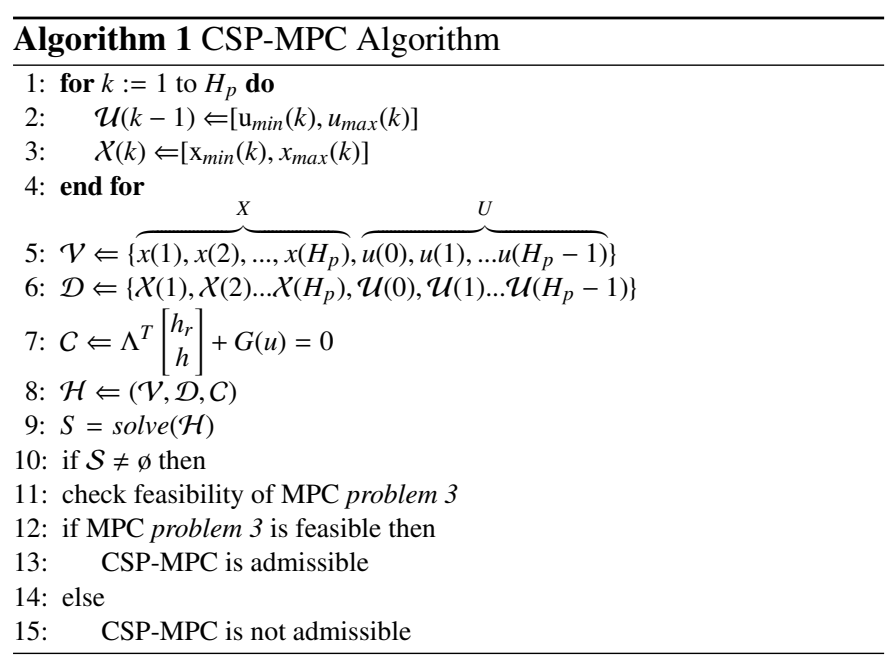

\section{CASE STUDY}

\subsection{Description}

To validate the proposed CSP-MPC approach, the Richmond case study is used. This network is introduced in Arunkumar and Mariappan (2011) includes one reservoir, four tanks, seven pumps and some pipes and valves.

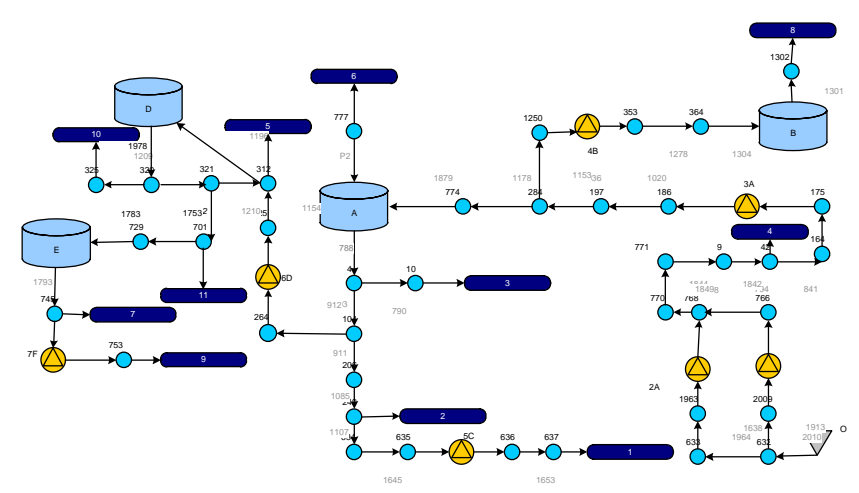

Figure 3. The Richmond water distribution system

\subsection{CSP for different configurations}

In Richmond distribution water network, there are mainly three different kinds of configurations, which lead to non-linear constraints in the MPC problem: 
Case 1 Valve Demand: demand connected to a tank by means of a valve.

Case 2 Pump Demand: demand connected to a tank by means of a pump.

Case 3 Complex Node Demand: demand connected with node, which has connections with more than one tank.

In this paper, only the Case 3 is presented due to space limitations.

Case 3: Complex Node Demand. One example for the configuration of complex node demand is shown in Fig. 4.

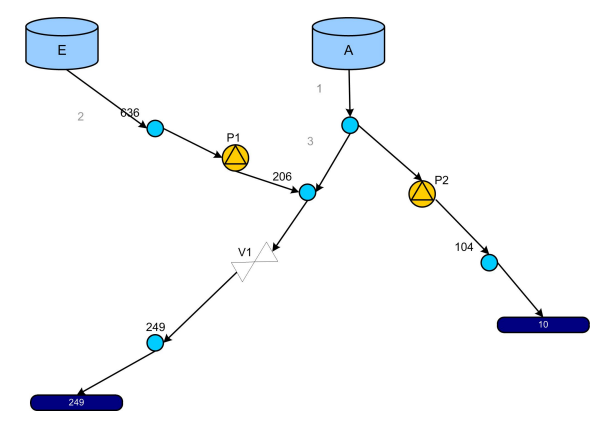

Figure 4. Complex Node Demand configuration

In this case, the CSP will be formulated in this way:

- $\mathcal{D}$ : Bounding Constraints, refer to physical limits

$$
\begin{aligned}
& u_{1} \in[0,10], u_{2} \in[0,50] \\
& u_{3} \in[0,30], x_{i} / \text { Sec }_{i} \in\left[0, X_{\text {max }_{i}}\right]
\end{aligned}
$$

- $C$ : Mass conservation constraints

$$
\begin{aligned}
x_{E_{1}} / S e c_{E} & =g_{p_{1}}+g_{p_{2}}+\Delta E_{1} . \\
x_{E_{2}} / S e c_{E} & =g_{p_{3}}+\Delta E_{2} . \\
x_{E} / S e c_{E} & =\max \left(x_{E_{1}}, x_{E_{2}}\right) \\
x_{A_{1}} / \operatorname{Sec}_{A} & =g_{p_{4}}+g_{p_{5}}+\Delta E_{3} . \\
x_{A_{2}} / S e c_{A} & =\operatorname{sum}\left(g_{p}\right)+\Delta E_{4} . \\
x_{A_{3}} / S e c_{A} & =\operatorname{sum}\left(g_{p}\right)-g_{b_{1}}+\Delta E_{5} . \\
x_{A_{4}} / S e c_{A} & =\operatorname{sum}\left(g_{p}\right)-g_{b_{2}}+\Delta E_{6} . \\
x_{A} / S e C_{A} & =\max \left(\left[x_{A_{1}}, x_{A_{2}}, x_{A_{3}}, x_{A_{4}}\right]\right)
\end{aligned}
$$

After running CSP:

- $\mathcal{H}$ : Updated constraints

$$
\begin{aligned}
& u_{1} \in[3.4,10], u_{2} \in[2.3,50] \\
& u_{3} \in[1.5,30], x_{A} \in[10,43] \\
& x_{E} \in[4.5,30]
\end{aligned}
$$

\subsection{Results}

Results of CSP-MPC. Through using CSP, Problem 2 has been transformed into Problem 3 by updating constraints for both tanks and also actuators. Fig. 5 shows evolution of real tanks volume comparing with its updated minimal constraints, which has been produced by CSP for satisfying pressure in every demand node. As shown from the plots, the added constraints for tanks affect water volume, which guarantee pressure for the demand node.

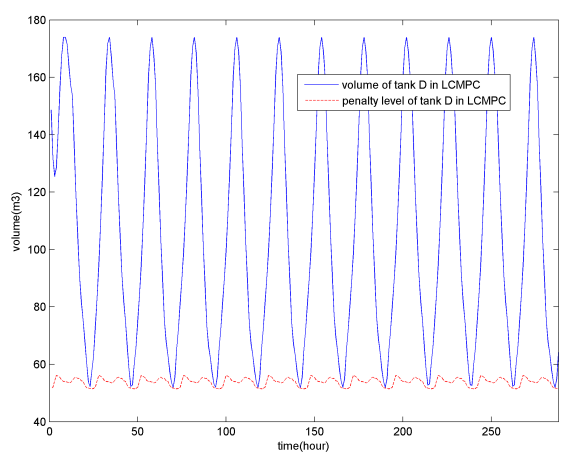

Figure 5. Water evolution of tankD

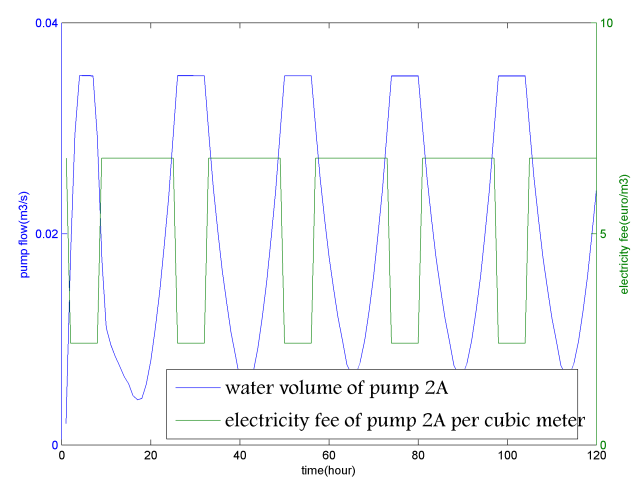

Figure 6. Pump flow with electricity price

The MPC-CSP should minimize costs associated to water transportation when pumping water from lower elevation to the higher elevation. In order to show how electrical cost optimization works, the case of pump $2 A$ will be used as an illustrative example. Fig. 6 shows in the same plot the pump flow and the electricity fee. From this figure, it can be noticed that the pump sends more water at the lower price period and less or no water at the higher price period.

\subsection{Validation of CSP-MPC using PLIO}

PLIO, which is a tool for the optimal operational control of DWN that implements non-linear MPC as introduced in Cembrano et al. (2011), has been developed using standard GUI(graphical user interface) techniques and objective oriented programming using Visual Basic.NET. In PLIO, models are built using the GAMS optimization modelling language. The resulting non-linear optimization problem is solved using CONOPT, which is a solver for large-scale nonlinear optimization problem (NLP) and is developed and maintained by ARKI Consulting Development in Denmark. CONOPT is a feasible path solver based on the proven GRG method as in Flores-Villarreal and Rios-Mercado (2003) with many newer extensions. All components of CONOPT have been designed for large and sparse models with over 10,000 constraints. In this paper, PLIO has been used as a validation of the CSP-MPC control scheme. 
Table 1. Compar. betw. PLIO and CSP-MPC

\begin{tabular}{llll}
\hline $\begin{array}{l}\text { Define } \\
\text { Name }\end{array}$ & \multicolumn{1}{c}{ PLIO } & \multicolumn{1}{c}{ CSP-MPC } & Improvement \\
\hline $\mathbf{J}_{\text {cost }}$ & 153.04 & 157.46 & $2.89 \%$ \\
$\mathbf{J}_{\text {smothness }}$ & 16.65 & 18.20 & $9.31 \%$ \\
$\mathbf{J}_{\text {safety }}$ & 0 & 0 & $0 \%$ \\
Comput. time & 400 & 140 & $-185.71 \%$ \\
\hline
\end{tabular}

Comparison Results. With the CSP-MPC control scheme, both linear and non-linear constraints of DWNs should be satisfied and optimal solution produced by CSP-MPC should be similar with that from PLIO

Fig. 7 and Fig. 8 show comparisons between CSP-MPC and PLIO on pump flows and pressure at demand nodes, which validate the feasibility of the CSP-MPC control structure for non-linear pressure problems of DWNs.

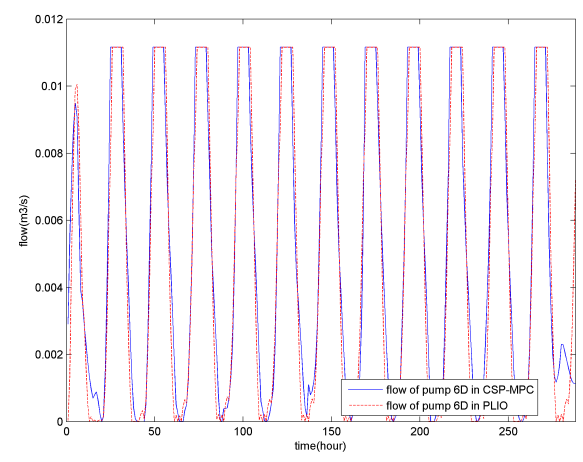

Figure 7. Pump flow comparisons

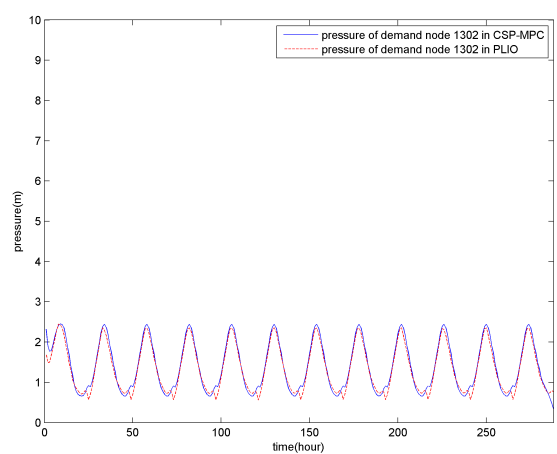

Figure 8. Demand node pressure comparisons

Table 1 shows the comparisons between PLIO and CSP-MPC in operational goals as analyzed in Section 3. The indices representing costs are given in economic units (e.u.) due to confidentiality restrictions. The row of Comput. time compared the needed computing time between PLIO and CSP-MPC and the time unit is minute. The column of Improvement is the improved proportion of results from PLIO to the CSP-MPC control.

\section{CONCLUSIONS}

This paper verifies linear MPC methodology for solving nonlinear problem in DWNs with the cooperation of CSP. The methodology successfully controls non-linear pressure model at DWNs in a linear way. Richmond water distribution network has been used as the case study and PLIO has been used to compare and verify for the results, while Epanet has been used as the water network simulator. Results comparison between PLIO and CSP-MPC verifies that the CSP-MPC control scheme is not only feasible but also computation time superior to the complex non-linear hydraulic problem in DWNs.

\section{ACKNOWLEDGEMENTS}

This research has been partially funded by the DGR of Generalitat de Catalunya (SAC group Ref. 2009/SGR/1491), Doctorat Industrial 2013-DI-041 and by EFFINET.

\section{REFERENCES}

AbdelMeguid, H.S. (2011). Pressure, Leakage and Energy Management in Water Distribution Systems. Phd thesis, De Montfort University.

Arunkumar, M. and Mariappan, V.N. (2011). Water demand analysis of municipal water supply using epanet software. International Journal on Applied Bioengineering, 5, 9-19.

Brdys, M. and Ulanicki, B. (1994). Operational Control of Water Systems: Structures, algorithms and applications. Prentice Hall International, United States.

Cembrano, G., Quevedo, J., Puig, V., Pérez, R., Figueras, J., Verdejo, J.M., Escaler, I., Ramón, G., Barnet, G., Rodríguez, P., and Casas, M. (2011). Plio: A generic tool for real-time operational predictive optimal control of water networks. Water Science and Technology, 64, 448-459.

Flores-Villarreal, J.H. and Rios-Mercado, Z.R. (2003). Computational experience with a grg method for minimizing fuel consumption on cyclic natural gas networks. Computational methods in circuits and systems applications, , 90-94.

Jaulin, L., Kieffer, M., Didrit, O., and Walter, E. (2001). Applied Interval Analysis, with Examples in Parameter and State Estimation, Robust Control and Robotics. Springer-Verlag, London.

Kumar, V. (1998). M. yokoo and e.h. durfee and t. ishida and k. kuwabara. IEEE Transactions on Knowledge and Data Engineering, 10, 673-685.

Maciejowski, J. (2002). Predictive Control with Constraints. Prentice Hall, Great Britain.

Ocampo-Martinez, C., Barcelli, D., Puig, V., and Bemporad, A. (2012). Hierarchical and decentralised model predictive control of drinking water networks: Application to barcelona case study. IET Control Theory and Applications, 6, 62-71.

Papageorgiou, M. (1983). Optimal control of generalized flow networks. System Modelling and Optimization Proc, 59, 373-382.

Rawlings, J. and Mayne, D. (2009). Model predictive control: theory and design. Nob Hill Publishing, Madison.

Tornil-Sin, S., Ocampo-Martinez, C., Puig, V., and Escobet, T. (2014). Robust fault diagnosis of non-linear systems using interval constraint satisfaction and analytical redundancy relations. IEEE Transactions on Systems, Man and Cybernetics, Part A: Systems and Humans, 44, 18-29.

Zessler, U. and Shamir, U. (1989). Optimal operation of water distribution systems. J. Water Resour. Plng. Mgmt., 115, $735-752$. 